\title{
Binaural hearing and time window in the transient
}

\author{
Yoshimasa Sakurai and Hiroshi Morimoto \\ Faculty of Engineering, Kansai University, \\ 3-3-35, Yamate-cho, Suita, 564 Japan
}

(Received 20 January 1989)

\begin{abstract}
It was reported that in the early stage of monaural hearing, the hearing system has an interfering transient response on loudness to the $0.05 \mathrm{~ms}$ rectangular pulse excitation and there the loudness to each pulse is added having its sign. However, at binaural hearing, the loudness of two pulses of different signs from the opposite lateral directions was summed in the absolute manner and it was almost the same as the loudness of two rectangular pulses in the same sign or a single pulse of double the amplitude from one lateral direction. Loudness of reflected sound fields caused by two different sized plane panels having the direct pulsive sound were compared. The loudness difference between them was not explained by the difference of the largest instantaneous loudness, but was done by the integration of the loudness amplitude in the time window. In the last, the transient process on the hearing system for the loudness of $0.05 \mathrm{~ms}$ rectangular pulse was summarized with new understandings in the earlier and present paper.
\end{abstract}

PACS number: 43.66. Mk, 43.66. Pn

\section{INTRODUCTION}

In an earlier paper, ${ }^{1)}$ the transient response on loudness of the hearing system to the $0.05 \mathrm{~ms}$ rectangular pulse was measured, and it was presumed as the contributions from the wave and elastic reactions in the initial stage of the system, which might include even the early part of the peripheral nerve. Here as well as in the earlier paper, loudness is used for the temporal perception to a pulse having a positive or negative sign and not used on the traditional definition of "sone." When it is expressed on a gragh, it is labeled by loudness magnitude not to be confused with the traditional definition. In this paper, the transient response on loudness in the later stage to the rectangular pulse was experimentally observed and the behavior of the hearing system was summerized.

In order to see how the sign of each pulse from different directions acts to have their loudness at binaural hearing, the loudness of two pulses of the same sign or different signs was asked when they arrive at the same time from the opposite lateral directions.

In the real sound field of a direct and a reflected sound from a small or a large rigid plane panel, which yields large or small negative boundary waves, ${ }^{2)}$ respectively, it was observed how the loudness between them is different.

The rectangular pulse used was obtained in the same way as in the previous papers. ${ }^{1,3-5)}$ A rectangular pulse of $0.05 \mathrm{~ms}$ in length was recorded on the PCM tape recorder at first. It was reproduced by the full range type loud-speaker in the anechoic chamber and picked up by a condenser microphone placed $1.2 \mathrm{~m}$ in front of the loud-speaker to measure the transfer function of the system. The inverse filter of this transfer function was then used in the loud-speaker circuit to produce a rectangular pulse at the receiving point $1.2 \mathrm{~m}$ in front of the loud-speaker. This was achieved by convolving the filter with a rectangular pulse on the desk top computer, the result then being converted to an analog signal, and recorded on the PCM recorder to be reproduced by the same loudspeaker. The signal was also convolved with a 
Hanning window to avoid problems with rise times. The final pulse spectrum used for the experiments has steep decreasing slope over $10 \mathrm{kHz}$. However, the frequency characteristics of the transient response obtained in the previous paper shows less sensitive in the range and is expected not to affect the experiments here. The amplitude of the rectangular pulse used was $93 \mathrm{~dB}$ referred to the steady level of the piston-phone. The male students used as subjects for the experiments were tested by audiometry and proved to be audiologically normal.

\section{BINAURAL HEARING AT THE TRANSIENT ${ }^{4)}$}

The transient response discussed in the earlier paper was measured for sources directly in front of the listeners. The transient response obtained, although measured with binaural hearing, will be essentially that for monaural hearing since each ear received the same signal at the same time. In order to see the form of the interference response for a more truly binaural hearing situation, the positive and negative rectangular pulses having the same amplitude were presented simultaneously to the respective ears for directions of \pm 90 degrees (see in Fig. 1).

When an omnidirectional microphone received the two pulses at a location equal to the mid-point between the two ears, the signals were naturally cancelled. However, when heard binaurally there, the loudness was much larger than that of a single rectangular pulse. Then, the loudness of binaural hearing of a positive and a negative pulse to each ear in Fig. 2(a), was compared by four male students, in the pair of two cases in Fig. 2(b) and (c), respectively. It was a loudness similar to that perceived with binaural hearing when two rectangular pulses

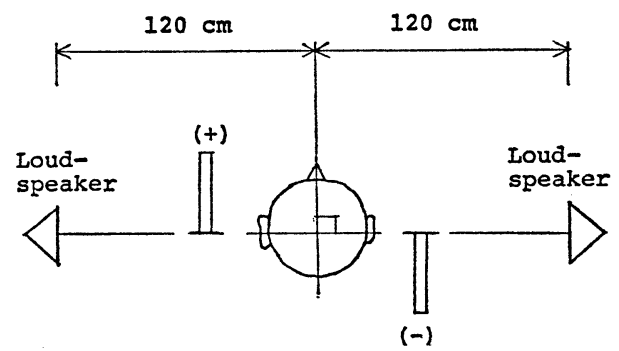

Fig. 1 A positive and a negative rectangular pulse of $0.05 \mathrm{~ms}$ length from the opposite lateral directions. having the same sign were presented from the loudspeakers. It was also similar to the loudness of a single pulse with double the amplitude from the one direction.

This fact tells us that, at the level in the hearing system where binaural effects occur, the signs of the transmitted signals are irrelevant and their resulting loudness corresponds to an adddition of their amplitudes. The reason why there appeared to be small difference between the loudness of a single pulse with double the amplitude and the loudness of two pulses either with the same or different sign is felt to result from the effects of a diffracted pulse from the opposite side of the head interfering with the input to each ear.

A difference in the localization between two pulses with different signs compared with two of the same sign was also noticed in the vertical plane. The former localization was slightly lower than the latter.

When the transfer function from a point source to a receiving point in an auditorium is measured with an omnidirectional microphone, the impulse response records the effects after interference between positive and negative reflections from the boundaries in the auditorium. Judging from our results, the method does not correspond to our way of hearing

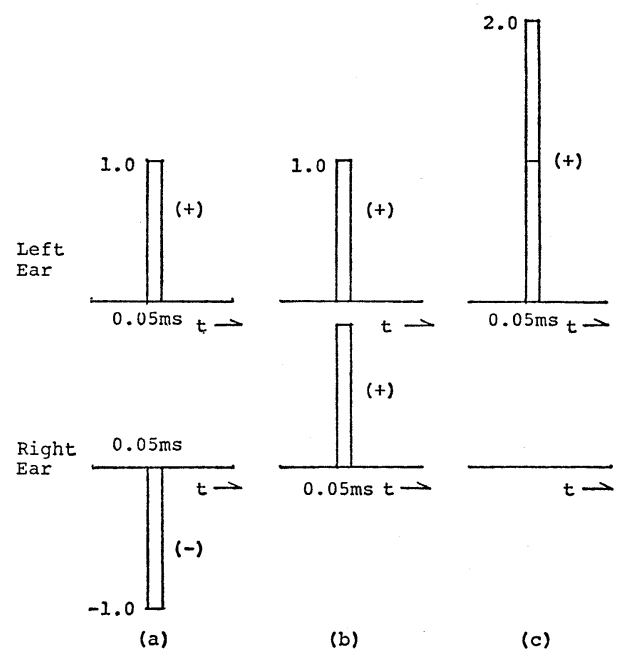

Fig. 2 Pair comparison of binaural hearing; (a) a positive and a negative pulse to each ear, (b) a positive pulse to each ear and (c) a pulse with double the amplitude to one ear. The amplitude 1.0 corresponds to $93 \mathrm{~dB}$. 


\section{Y. SAKURAI and H. MORIMOTO: BINAURAL HEARING AND TIME WINDOW}

the reflected sound field binaurally, as well as losing the spatial information in the sound field.

Previously, the technique referred to as "Visual Sound Field"6) was introduced to show the spatial information in the impulse response of a sound field. For a better acoustical estimation of an auditorium its impulse response should be convolved with the ear's transient responses.

\section{INTEGRATION IN THE TIME DOMAIN FOR CONTINUOUS SIGNALS ${ }^{3,4)}$}

In order to investigate the effect of negative boundary waves in real sound fields for the hearing system, a pulse from a loud-speaker together with its reflection from small and large rigid square plane panels whose side lengths are $45 \mathrm{~cm}$ and $90 \mathrm{~cm}$, respectively, as shown in Fig. 3 was generated. The sound fields measured by an omnidirectional half inch condenser microphone (B \& $\mathrm{K}$, type 4134) are shown in Fig. 4(i) and (ii). A $20 \mathrm{~mm}$ thick fiber glass cover was used to suppress the reflection of a panel. As the loud-speaker generated the $0.05 \mathrm{~ms}$ rectangular pulse $1.2 \mathrm{~m}$ in front of it with the amplitude of $93 \mathrm{~dB}$ and faced equally to the receiving point and the center of each panel, the edge wave of the loud-speaker's diaphragm produced directivities and the direct pulse has following continuous waves. The specular reflection from the small panel in Fig. 4(i) is followed by large negative boundary waves.

The transient response of interference, the average twelve male students, which is shown in Fig. $5^{1)}$ was convolved with the recorded sound fields and the results are shown in Fig. 6, though the four male students who judged the differences among three sound fields were not included in the twelve

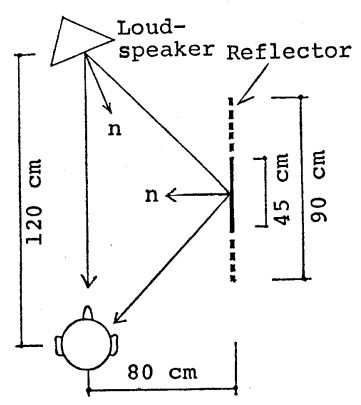

Fig. 3 Reflection off a small or a large rigid plane square panel. male students whose average transient response is shown in Fig. 5. Figure 6(i) shows its convolution with the direct sound, Fig. 6(ii) shows that with the direct sound and the reflection from the small rigid panel and Fig. 6(iii) shows that with the direct and its reflection from the large panel. More rigorously, the directional hearing transient reponse, which is related to the directivity of the head related trans-

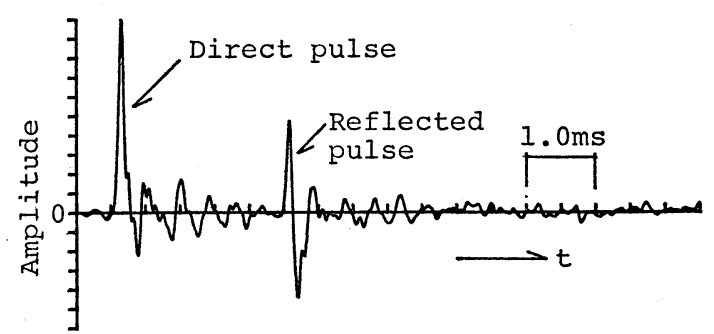

(i) Direct pulse and a reflection from a small rigid plane panel.

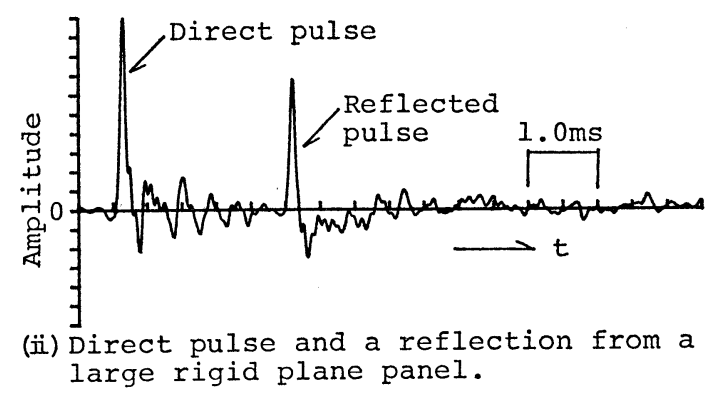

Fig. 4 Sound fields reflected by different sized rigid square plane panels.

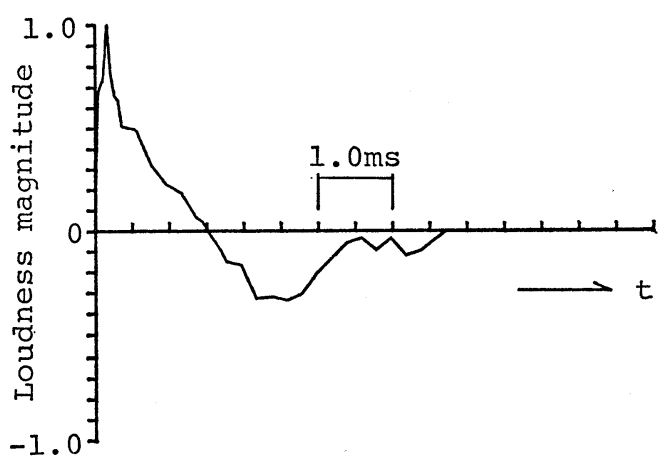

Fig. 5 Transient response of interference of the human hearing system to a rectangular pulse of $0.05 \mathrm{~ms}$ length and $93 \mathrm{~dB}$ amplitude. It is the average value for twelve male students. 


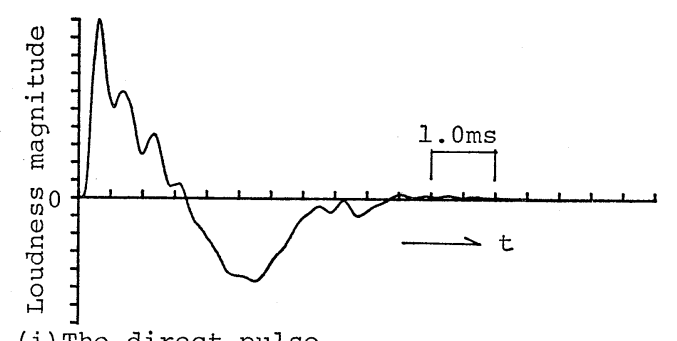

(i) The direct pulse.

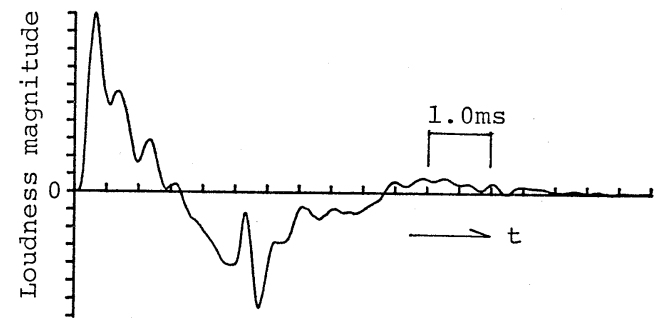

(ii) The direct pulse and the reflection from the small rigid plane panel.

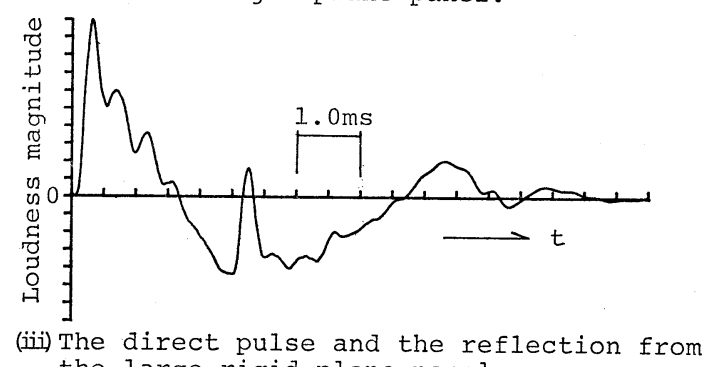
the large rigid plane panel.

Fig. 6 Instantaneous loudness magnitude of three sound fields obtained by the convolution of the averaged transient response in Fig. 5.

fer function, should be convolved with each reflection, but it does not make much difference in this case. The effect of the reflection from the small rigid panel on the temporal loudness magnitude is not much recognized in the figure because of the large negative boundary waves.

When asked if the loudness was raised by the reflections from the panels, all of four male subjects noted an increase, but only for the case of the large panel. However, the difference between the highest temporal loudness magnitudes of three cases can not be seen in Fig. 6(i), (ii) and (iii).

With this time interval between the two pulses we would expect that they might be heard separately but they were not. The explanation is thought to be that, although the signals came from different directions, they are highly correlated still and are judged as a continuous signal. When information is obtained from continuous acoustical data, a few kinds of time windows are thought to cut out the period from the latest past to the present. ${ }^{7)}$ The loudness, which would correspond to the traditional expression, of the continuous acoustical signal must be obtained by the temporal integration of the instantaneous signal loudness over the window. The particular shape of the time window must be determined by the auto-correlation of the original acoustical signal. In this particular experiment the time window is thought to be long enough to include all of the reflected sound field. Hence an integration was performed over the absolute value of the instantaneous singnal loudness. When the direct pulse thus integrated is the reference, the loudness levels with the reflection off the small rigid panel and off the large one were raised by $0.7 \mathrm{~dB}$ and $2.3 \mathrm{~dB}$, respectively. In the latter case the loudness level increase is sufficient that it is possible to hear a raising of the loudness by the reflection.

For a long continuous acoustical signal, a running time window should be introducted. The relation between the auto-correlation of a sound signal and the effective time window should be the subject of further investigation.

\section{OUTLINE OF THE HUMAN HEARING SYSTEM OPERATION AS SUGGESTED BY THE PRESENT RESULTS}

From the results in this and the earlier papers, the response of our hearing system is imagined as follows. First, the linear loudness transient response of the ear from the pinna to the basilar membrane which also may include a beginning part of the peripheral neural system is convolved with an input signal. Secondly, the absolute values of the magnitude of the instantaneous loudness are obtained before processing of the binaural signals occurs. Some selection and classification occurs based on the type of signal, and the selected and classified signals are given dedicated pathways. For instance, pure tones do not beat with low pitch sounds and the resonance frequency of the external meatus suffers some smoothing as discussed in the earlier paper, pure tone pathways look different from those via which the loudness of a rectangular pulse is processed. The loudness of the classified signal is then determined by an integration over a time window, 


\section{Y. SAKURAI and H. MORIMOTO: BINAURAL HEARING AND TIME WINDOW}

the length of which depends in some way on its information content and also the auto-correlation function of the signal. This window extends from the present to the nearest past. The non-linear filter which gives the power or logarithmic law demonstrated by the ear is caused by a saturation process occurring at signal extremes and limited at the other end by the inner noise resulting from normal human activity.

\section{CONCLUSIONS}

After a sound which excites the ear is convolved with the transient response of the peripheral monaural hearing system, the signal is transmitted in an absolute form and, at the stage of binaural processing, the signals from the two ears are added in their absolute forms. When two rectangular pulses with different signs were heard binaurally from the opposite lateral directions at the same time, they were added in the absolute forms and were heard similar to the loudness of two positive pulses from the opposite lateral directions or the single pulse of double the amplitude from one lateral direction. The localization of two pulses from the opposite lateral directions were a little different in the vertical plane depending on their signs.

For a continuous acoustical signal, a time window for temporal integral is required.

\section{ACKNOWLEDGEMENTS}

The authors are grateful to Dr. George Dodd at the Acoustics Institute, the University of Auckland, for his discussions and help in English translations.

\section{REFERENCES}

1) Y. Sakurai and H. Morimoto, "Transient response of human hearing system," J. Acoust. Soc. Jpn. (E) 10, 221-228 (1989).

2) Y. Sakurai and K. Nagata, "Sound reflections of a rigid plane panel and of the 'live end' composed by those panels," J. Acoust. Soc. Jpn. (E) 2, 5-14 (1981).

3) Y. Sakurai, "Transient response of human hearing system," Proc. 12th ICA, Toronto, B3-1 (1986).

4) Y. Sakurai and H. Morimoto, "Transient response of human hearing system," Tech. Rep. Hearing Acoust. Soc. Jpn. H-87-28 (1987) (in Japanese).

5) Y. Sakurai and H. Morimoto, "Transient response of human hearing system (part 2)," Tech. Rep. Hearing Acoust. Soc. Jpn. H-87-65 (1987) (in Japanese).

6) Y. Sakurai, "Visual sound field," Proc. 11th ICA, Paris, Vol. 4, 437-440 (1983).

7) I. J. Hirsh, "Temporal aspects of hearing," in The Nervous System, Vol. III Human Communication and Its Disorders, E. L. Eagles, Ed. (Raven Press, New York, 1975), pp. 157-162. 